\title{
Molecular field theory for polar, biaxial bent-core nematics
}

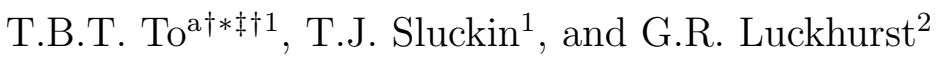 \\ ${ }^{1}$ Mathematical Sciences, University of Southampton, , Southampton, SO17 1BJ, \\ United Kingdom \\ ${ }^{2}$ Chemistry, University of Southampton, , Southampton, SO17 1BJ, United \\ Kingdom
}

March 42016

\begin{abstract}
We have studied a polar, biaxial nematic liquid crystal formed from bent-core molecules using molecular field theory. The model includes a simple Heisenberg-form dipolar intermolecular interaction in addition to the usual quadrupolar nematic interaction, and mimics a system consisting of nematogenic bent-core molecules with an large transverse dipole along the bisector of the two molecular arms. Such systems are regarded as good candidates for biaxial nematic liquid crystals. In principle, the molecular dipoles can align, thus stabilizing the ordering of the minor axes. Our calculations predict that, for suitable values of the bent-core interarm angle, the biaxial nematic phase can be stabilized at higher temperatures than in the absence of the transverse dipole. In general, the transverse macroscopic polar order stabilizes the biaxial nematic phase. In particular, for a large enough dipolar interaction, the Landau point in the pure biaxial nematic develops into a line of first-order polar biaxial nematic-to-isotropic phase transitions.
\end{abstract}

\section{Introduction}

Most nematic liquid crystals exist as uniaxial phases $\left(N_{U}\right)$. An idealized picture of these phases is such that constituent molecules align one molecular

\footnotetext{
${ }^{* \dagger}$ Corresponding author. Email: tung.to@univ-lyon1.fr

${ }^{\dagger}$ Institut Lumière Matière, UMR5306 Université Lyon 1-CNRS, Université de Lyon, 69622 Villeurbanne, France
} 
axis to form a single macroscopic director. Over forty years ago, Freiser [?] predicted the possible existence of a biaxial nematic liquid crystal phase $\left(N_{B}\right)$. In this phase, all three molecular axes align to form three macroscopic directors. In the intervening period the subject has been the focus of much intensive theoretical (e.g. [?, ?, ?, ?]), computational (e.g. [?, ?]) and experimental (e.g. [?, ?, ?, ?]) research.

There are well-attested observations of lyotropic biaxial nematic phases [?]. There have also been well-publicised reports of the existence of thermotropic biaxial nematic phases in bent-core nematics [?, ?]. But this work has attracted some controversy in the literature (see e.g. [?, ?, ?]). In particular, the onset of the experimental $N_{B}[?, ?]$ seems to occur at a much higher temperature than that predicted theoretically [?] and the phase biaxiality is very small. It has been suggested [?, ?] that this may be due to a large permanent tranverse electrostatic dipole in the bent-core molecules. Electro-optical experiments by Lee et al.[?] have shown some evidence of this phase polarity. However if this were true, the resulting phase might be expected to be polar, due to the ordering of the molecular dipoles [?]. This paper concerns the possibility of polar nematics formed from bent-core molecules with transverse electrostatic dipoles.

The possibility of a ferroelectric biaxial nematic phase was discussed long ago by Palffy-Muhoray et al.[?]. By using a molecular field theory for ferroelectric uniaxial nematics, the authors concluded that transverse dipoles on rod-like molecules tend to align parallel and this might lead to a ferroelectric biaxial nematic. Other studies using molecular field theory by Sonnet et al.[?, ?], which include both electrostatic interactions and steric effects, predict that although electrostatic interactions promote parallel alignment of electrostatic dipoles, polar steric effects favour antiparallel alignment of bent-core molecules. Thus within this theory [?, ?], polar nematic phases will be formed if the electrostatic interactions were to be large enough to overcome steric effects.

We recall the potentially interesting effect of the macroscopic polarisation of a nematic phase on the director distribution. Many years ago Meyer [?] showed that a polarization would produce a flexopolarization coupling, causing the uniform nematic to be unstable with respect to a chiral phase of indeterminate sense. In this chiral phase - the twist-bend nematic phase - the director is tilted with respect to the helix axis of the chiral phase. This prediction long lay essentially unrecognised, but interest in this novel nematic phase was reawakened largely by the prediction of Dozov [?] that bent molecules can also form such a phase. The bend in the molecule significantly reduces the bend elastic constant, stabilizing the twist-bend nematic. 
It was this prediction that prompted the discovery of the twist-bend nematic phase for liquid crystal dimers having odd spacers [?]. It remains to be seen, however, which of these predicted driving mechanisms are responsible for the existence of the twist-bend nematic phase [?].

The effect of the electrostatic dipolar interactions in bent-core molecules has been studied using lattice Monte Carlo simulations by Bates [?] and Ghoshal et al.[?]. In the former work, the dipolar interaction is modeled by a Heisenberg form which only depends on the relative orientation of two interacting molecules. In the latter work, which assumes the same secondrank quadrupolar interaction, the dipolar interaction is more comprehensive and also depends on the relative position of the two interacting molecules. No polar phases were found in either study, in agreement with a density functional theory developed by Grzybowski and Longa [?], a series of atomistic simulations by Peláez and Wilson [?] and a two-particle-cluster theory by Osipov and Pająk [?]. However, by contrast, Ghoshal et al.[?] suggested that the unrealistic Heisenberg form of dipolar interaction potential used by Bates [?] might form polar phases. Moreover, they asserted that long-range directional contributions are unfavourable to dipolar phase.

This paper addresses the apparent contradiction between these points of view. We develop a molecular field theory with an internal energy analogous to the pair potential used in the lattice simulations by Bates [?], with the specific aim of investigating whether this interaction potential can form polar nematics. The paper is organized as follows. In Section ?? we derive our molecular field theory from the pair potential used by Bates [?]. In Section ?? we present results from our calculations where we use the same parameter values as Bates [?]. Finally in Section ?? we discuss the context in which our results should be understood.

\section{Molecular field theory}

\subsection{Basis functions and order parameters}

The molecular field theory requires an orientational distribution function $f(\Omega)$ and a set of order parameters. A complete theory for biaxial nematics $C_{2 v}$ symmetry (that of the letter $\mathrm{V}$ ) formed from molecules with $C_{2 v}$ symmetry requires a first-rank order parameter and four second-rank order parameters. These second-rank order parameters are the same as those with $D_{2 h}$ symmetry. They are denoted as $(S, D, P, C)[?, ?]$, which are orientational averages of basis angular functions $\left(R_{S}, R_{D}, R_{P}, R_{C}\right)$. We follow the notation convention of Teixeira et al.[?], but with different scaling (see also 
Dunmur and Luckhurst [?]). The functions are:

$$
\begin{gathered}
R_{S}(\Omega)=\frac{1}{2}\left(3 \cos ^{2} \beta-1\right) ; \\
R_{D}(\Omega)=\sqrt{\frac{3}{8}} \sin ^{2} \beta \cos 2 \gamma ; \\
R_{P}(\Omega)=\sqrt{\frac{3}{8}} \sin ^{2} \beta \cos 2 \alpha ; \\
R_{C}(\Omega)=\frac{1}{2}\left(1+\cos ^{2} \beta\right) \cos 2 \gamma \cos 2 \alpha \\
-\cos \beta \sin 2 \gamma \sin 2 \alpha .
\end{gathered}
$$

The order parameters are defined in terms of basis angular function averages:

$$
i=\left\langle R_{i}\right\rangle=\int d \Omega f(\Omega) R_{i}(\Omega) .
$$

with $i \in\{S, D, P, C\}$. We note that (subject to exchange of axes) in the $N_{U}$ phase, in general, $S, D \neq 0$, but $C=P=0$. In biaxial phases all four order parameters in general are non-zero. By convention, for the ground state, $S=C=1$ and $D=P=0$. We also note, as discussed by a number of authors (see e.g.[?]), when the molecular axes $(x, y, z)$ are permuted, the order parameters $\{S, D, P, C\}$ are subject to linear transformation.

Our molecular field theory for polar biaxial nematics requires a firstrank order parameter, in addition to the four second-rank order parameters $(S, D, P, C)$. This is the orientational average of the basis function $R_{1}(\Omega)$. The exact form for $R_{1}$, as for the second-rank order parameters $\{S, D, P, C\}$, depends on our labeling of molecular axes $(x, y, z)$. The precise formulae are presented in section ?? (see Eqs. (??),(??),(??)). The first-rank order parameter is defined in term of the basis angular function average

$$
\mathcal{P}=\left\langle R_{1}\right\rangle=\int d \Omega f(\Omega) R_{1}(\Omega) .
$$

\subsection{Basic material}

In this paper, the molecular orientation $\Omega$ is parameterized in terms of the three Euler angles $\alpha, \beta, \gamma$ which take their conventional meanings (polar angle $\beta$ and zenithal angles $\alpha, \gamma$ ). The Euler angle $\alpha$ is only of interest 
in the spontaneously biaxial phases. We suppose the constituent bentcore molecules possess $C_{2 v}$ symmetry. Thermodynamic quantities, namely the free energy, internal energy, potentials of mean torque, orientational distribution functions and partition functions are formulated per particle. In addition, the temperature and energy quantities are expressed in nondimensional units. We denote the internal energy by $U$, and the potential of mean torque acting on a molecule with orientation $\Omega$ by $U(\Omega)$. In addition, $\epsilon$ denotes the energy scaling parameter which scales the interaction between a uniaxial arm and the molecular field.

Our molecular field theory relies on the Maier-Saupe theory of nematic ordering (see e.g. de Gennes and Prost [?]) for which the free energy per particle is given by:

$$
A=k_{B} T \int_{\Omega} d \Omega f(\Omega) \ln 8 \pi^{2} f(\Omega)-\frac{1}{2} u S^{2},
$$

where $S$ is the nematic order parameter, defined as the average of the secondrank Legendre function

$$
S=\left\langle P_{2}(\cos \beta)\right\rangle=\left\langle\frac{3 \cos \beta^{2}-1}{2}\right\rangle,
$$

where $k_{B}$ denotes the Boltzmann constant, $T$ is the absolute temperature and $u$ sets the scale of the molecular anisotropic interaction.

The value of the molecular interaction scale $u$ (see Eq. (??)) depends on $\epsilon$, the molecular interarm angle $\theta$ and the molecular coordinate axes set in the molecule. This dependence will be derived in Sections ?? and ??. We shall work, where possible, in non-dimensional quantities, given in terms of their physical quantities by

$$
\begin{aligned}
T^{*} & =\frac{k_{B} T}{\epsilon} ; \quad A^{*}=\frac{A}{\epsilon} ; \\
U^{*} & =\frac{U}{\epsilon} ; \quad U^{*}(\Omega)=\frac{U(\Omega)}{\epsilon} .
\end{aligned}
$$

The theory combines elements of the molecular field theory of biaxial nematics with $D_{2 h}$ symmetry (that of a rectangular parallelepiped) developed elsewhere $[?, ?, ?, ?]$ with the molecular field theory of polar uniaxial nematics [?]. At second-rank level, the theories for molecules formed from $D_{2 h}$ and $C_{2 v}$ symmetry are equivalent. We note that in this sense the mathematical structure of our model is equivalent to a system consisting of parallelepiped molecules each possessing a Heisenberg dipole. 


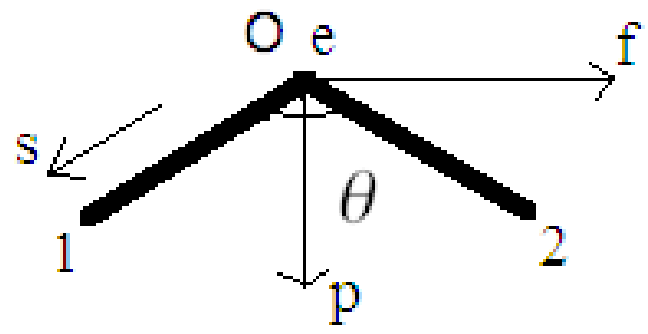

Figure 1: A sketch of our idealized bent-core molecule. $(p, e, f)$ are dummy labels of the molecular axes which can take any one of the permutations of $(x, y, z)$ (see Appendix ??).

\section{$2.3 \quad$ Lattice model}

We study the same molecular model as Bates [?]. In this model, bentcore molecules are composed of two identical uniaxial arms joined at one end with the interarm angle $\theta$ (see Fig. 1). For now we use the dummy variables $(p, e, f)$ to label the molecular axes. Thus $(p, e, f)$ can be any of the permutations of $(x, y, z)$. We label two interacting molecules as $A$ and $B$ and the two arms of a molecule as 1 and 2. This model is an adaptation of the Maier-Saupe model which neglects the dependence of pair interactions on the intermolecular vector. Following Bates [?], we suppose that two neighbouring point molecules interact via a pair potential

$$
\begin{array}{r}
U_{(2)}^{*}(\Omega)=\frac{U_{(2)}(\Omega)}{\bar{\epsilon}}=-\sum_{i, j=1,2} P_{2}\left(\cos \phi_{i j}^{A B}\right) \\
-\kappa \mathbf{p}_{A} \cdot \mathbf{p}_{B} .
\end{array}
$$

Here we use the subscript (2) to avoid confusion between the pair potential and the potential of mean torque (see Eq. (??)). $\bar{\epsilon}$ scales the interaction between two arms of two interacting molecules. $\phi_{i j}^{A B}$ denotes the angle between arm $i$ of molecule $A$ and arm $j$ of molecule $B$ and $\mathbf{p}_{k}$ is the unit vector along the $p$ axis of molecule $k=A, B$. Here $\Omega$ denotes the three Euler angles between the coordinate axes $(p, e, f)$ of molecules $A$ and $B$. The first term is the sum of the Maier-Saupe interactions of the uniaxial molecular arms. Although this is the sum of uniaxial interactions, when the interarm angle is different from $\left(0^{\circ}, 90^{\circ}, 180^{\circ}\right)$, the sum results in a biaxial interaction potential [?]. For two molecules interacting via a biaxial potential, at the ground state all three molecular axes align. In contrast, for two molecules 
interacting via a uniaxial potential, only one molecular axis aligns in the ground state. The second term is a Heisenberg model and is simpler than the electrostatic dipole-dipole interaction presented by Ghoshal et al.[?]

$$
U_{\mu}=-\psi\left(\mathbf{p}_{A} \cdot \mathbf{p}_{B}-3\left(\mathbf{p}_{A} \cdot \mathbf{r}\right)\left(\mathbf{p}_{B} \cdot \mathbf{r}\right)\right),
$$

where $\psi$ is a scalar; $\mathbf{r}$ denotes the intermolecular vector.

Osipov and Pajak [?] have shown that, subject to the absence of screening, the volume integral of the electrostatic dipole-dipole interation in Eq. (??) can be expressed as two terms. The first contribution to the potential has the Heisenberg form (the second term in Eq. (??)) and the second contribution depends on the boundary conditions. As an example, the dipole-dipole interaction is equal to the Heisenberg-like potential within the molecular field theory for a spherical sample in a conducting medium. In addition, the dipolar potential also has a non-zero Heisenberg form when the electrostatic dipole-dipole interaction is coupled to the anisotropic molecular shape of short-range correlations [?]. Thus our model can be considered as valid in those conditions.

Note that in our model, for $\kappa>0$, the state in which molecular dipoles align parallel has the lowest dipolar energy, which is only an idealization of dipolar interactions. The first-rank orientational function (see Eq. (??)) is defined by

$$
R_{1}(\Omega)=\mathbf{p}_{A} \cdot \mathbf{p}_{B} .
$$

In the simulations by Bates [?], each molecule interacts with its six nearest neighbours in a cubic lattice via the pair potential in Eq. (??). The temperature in the simulations is scaled with $\bar{\epsilon}$ (see Eq. (??)) whereas that in the molecular field theory is scaled with $\epsilon$ (see Eqs. (??)), the interaction between a rod and the director. Thus when comparing results from the two approaches, the temperatures calculated from the molecular field theory need to be multiplied by six.

Bates and Luckhurst [?] have shown that the second-rank interactions of the pair potential of bent-core molecules in Eq. (??) is equivalent to the pair potential of parallelepiped molecules with the separability approximation for the interaction tensors [?]

$$
U_{(2)}^{*}(\Omega)=\frac{U_{(2)}(\Omega)}{\epsilon_{m}}=-\left(J_{1}(\Omega)+2 \lambda J_{2}(\Omega)\right),
$$

where $\epsilon_{m}$ scales the interactions between two molecules. The relation between $\bar{\epsilon}$ and $\epsilon_{m}$ and is the same as the relation between $\epsilon$ and $u$ (see 
Section ??). $J_{1}(\Omega), J_{2}(\Omega)$ are linear combinations of the basis functions $R_{i}, i \in(S, D, P, C)$ (see Eqs. (??),(??))

$$
\begin{gathered}
J_{1}(\Omega)=R_{S}(\Omega)+2 \lambda R_{D}(\Omega), \\
J_{2}(\Omega)=R_{P}(\Omega)+\lambda R_{C}(\Omega) .
\end{gathered}
$$

The parameter $\lambda$ measures the biaxiality of interacting molecules, either in board-like or bent-core system. For bent-core molecules, $\lambda$ strictly depends on the interarm angle $\theta$ and their relation depends on our choice of the molecular axis systems.

\subsection{Mathematical model}

In order to map the second-rank interactions of bent-core molecules in Eq. (??) into Eq. (??), we have the option of selecting the set $(p, e, f)$ (see Fig. 1 ) from one of the permutations of $(x, y, z)$. Note that by convention our definition of the uniaxial order parameters are given by

$$
S=S_{z z}^{Z Z} ; \quad D=\frac{S_{x x}^{Z Z}-S_{y y}^{Z Z}}{\sqrt{6}},
$$

where, also by convention, $z$ is the molecular major axis which aligns in the uniaxial phase such that $S>D$, and $x$ and $y$ are labelled such that $D$ is positive.

There are thus three distinct cases, in which $z$ may correspond to $p, e$ and $f$, respectively. Previous studies by Ferrarini et al.[?] and Bates and Luckhurst [?] for pure second-rank interactions reported two of these cases. For $90^{\circ}<\theta \lesssim 109.5^{\circ}$, the molecule is discotic and the molecular axis $e$ aligns in the uniaxial phase. In this case, $e \equiv z$ and thus $(p, e, f) \equiv(y, z, x)$. For $109.5^{\circ} \lesssim \theta<180^{\circ}$, the molecule is calamitic and the molecular axis $f$ aligns in the uniaxial phase. In this case, $f \equiv z$ and thus $(p, e, f) \equiv(x, y, z)$. In our theory, when the dipolar interaction is strong enough, it can cause the molecular axis along $p$ to align to form a uniaxial nematic phase. In this case we need to define the molecular axis $p \equiv z$ and thus $(p, e, f) \equiv(z, y, x)$.

The detail derivations are given in Appendix ??. In all three cases, the pair potential can be written in a generalized form

$$
U_{(2)}^{*}(\Omega)=-g(\theta)\left(J_{1}(\Omega)+2 \lambda J_{2}(\Omega)\right)-\kappa R_{1}(\Omega) .
$$

The relation between the molecular energy scale (see Eq. (??)) and that of an arm is given by $\epsilon_{m}=g(\theta) \bar{\epsilon}$.

The dependence of $g(\theta), R_{1}(\Omega)$ and $\lambda(\theta)$ on $\theta$ is given by 
- Case 1: $(p, e, f) \equiv(x, y, z)$ rod-like molecule

$$
\begin{aligned}
& g(\theta)=\frac{1}{4}(1-3 \cos \theta)^{2}, \\
& \lambda=\sqrt{\frac{3}{2}} \frac{(1+\cos \theta)}{(1-3 \cos \theta)},
\end{aligned}
$$

and

$$
R_{1}(\Omega)=\mathbf{x}_{1} \cdot \mathbf{x}_{2}=\cos \beta \cos \gamma \cos \alpha-\sin \gamma \sin \alpha .
$$

- Case 2: $(p, e, f) \equiv(z, y, x)$ dipolar molecule

$$
\begin{aligned}
& g(\theta)=\frac{1}{4}(1+3 \cos \theta)^{2}, \\
& \lambda=\sqrt{\frac{3}{2}} \frac{(\cos \theta-1)}{(1+3 \cos \theta)},
\end{aligned}
$$

and

$$
R_{1}(\Omega)=\mathbf{z}_{1} \cdot \mathbf{z}_{2}=\cos \beta .
$$

- Case 3: $(p, e, f) \equiv(y, z, x)$ disc-like molecule

$$
\begin{gathered}
g(\theta)=1, \\
\lambda=-\sqrt{\frac{3}{2}} \cos \theta,
\end{gathered}
$$

and

$$
R_{1}(\Omega)=\mathbf{y}_{1} \cdot \mathbf{y}_{2}=\cos \gamma \cos \alpha-\cos \beta \sin \gamma \sin \alpha .
$$

The formulae for $\lambda$ in case 1 and 3 are in agreement with those given by Ferrarini et al.[?] and Bates and Luckhurst [?]. In Fig. 2 we show the mapping of $\theta$ to $\lambda$ for Case 1 (continuous line) and Case 3 (broken line). The dependence of $\lambda$ on $\theta$ for these two cases are shown in Fig. 2. The dotted broken horizontal line $\lambda=\sqrt{3 / 2}$ and the continuous line $\lambda=0$ mark the boundary of $\lambda$ that we discuss in this paper. The dotted broken horizontal line $\lambda=1 / \sqrt{6}$ marks the boundary between the rodlike and disc-like molecule in the $\lambda$ parameter space. Similarly, the vertical line $\theta=\theta_{c}=\cos ^{-1}(-1 / 3) \approx 109.5^{\circ}$ separates the rod-like and disc-like molecules in the $\theta$ parameter space.

First we discuss Case 1 , in which as $\theta$ increases from 0 to $180^{\circ}, \lambda$ decreases from $\sqrt{3 / 2}$ to 0 . For $\kappa=0$ and $0<\lambda<1 / \sqrt{6}\left(\theta_{c}<\theta<180^{\circ}\right)$, the molecule 


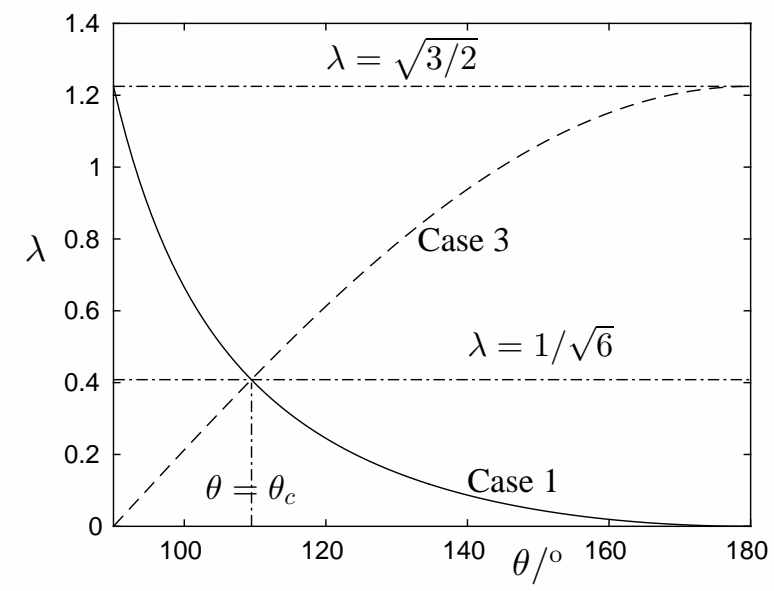

Figure 2: The biaxiality of bent-core molecules as a function of the interarm angle for Case 1 (continuous line) (Eq. (??)) and Case 3 (broken line) (Eq. (??)). $\theta_{c}=\cos ^{-1}(-1 / 3) \approx 109.5^{\circ}$. The dotted broken horizontal line $\lambda=\sqrt{3 / 2}$ and the continuous line $\lambda=0$ marks the boundary of $\lambda$ that we discuss in this paper. The dotted broken horizontal line $\lambda=1 / \sqrt{6}$ marks the boundary between the rod-like and disc-like molecule in the $\lambda$ parameter space. The vertical line $\theta=\theta_{c}=\cos ^{-1}(-1 / 3) \approx 109.5^{\circ}$ separates the rodlike and disc-like molecules in the $\theta$ parameter space. 
aligns along $z(\equiv f)$ in the $N_{U}$ phase. Since in this case the molecule is elongated, we identify the $N_{U}$ phase as rod-like, $N_{U}^{+}$. By contrast, for $\kappa=0$ and $1 / \sqrt{6}<\lambda<\sqrt{3 / 2}\left(90^{\circ}<\theta<\theta_{c}\right)$, the molecules align along $y(\equiv e)$ in the $N_{U}$ phase. Since in this case the molecule is oblate, we identify the $N_{U}$ phase thus formed as disc-like $N_{U}^{-}$.

Next we discuss Case 3 , as $\theta$ increases from 0 to $180^{\circ}, \lambda$ increases from 0 to $\sqrt{3 / 2}$. For $\kappa=0$ and $0<\lambda<1 / \sqrt{6}\left(90^{\circ}<\theta<\theta_{c}\right)$, the molecule aligns along $z(\equiv e)$ in the $N_{U}$ phase. Since in this case the molecule is oblate, we identify the $N_{U}$ phase formed as disc-like, $N_{U}^{-}$. By contrast, for $\kappa=0$ and $1 / \sqrt{6}<\lambda<\sqrt{3 / 2}\left(\theta_{c}<\theta<180^{\circ}\right)$ and, the molecules align along $y(\equiv f)$ in the $N_{U}$ phase. Since in this case the molecule is elongated, we identify the $N_{U}$ phase as rod-like $N_{U}^{+}$.

There is an equivalent transformation between Case 1 and Case 3 by changing the molecular axes. By changing the molecular axes from Case 1 to Case 3 , the region $1 / \sqrt{6}<\lambda<\sqrt{3 / 2}$ of the continuous line (Case 1 ) is mapped onto the region $0<\lambda<1 / \sqrt{6}$ of the broken line (Case 3 ). Similarly, by changing the molecular axes from Case 3 to Case 1, the region $1 / \sqrt{6}<\lambda<\sqrt{3 / 2}$ of the broken line (Case 3) is mapped onto the region $0<\lambda<1 / \sqrt{6}$ of the continuous line (Case 1 ).

For numerical calculations, it is more convenient to have $z$ as the molecular axis that aligns in the $N_{U}$ phase. Thus for $\kappa=0$ and $1 / \sqrt{6}<\lambda<\sqrt{3 / 2}$, we need to relabel the axes so that $0<\lambda<1 / \sqrt{6}$. Namely, if we are using Case 1 then we need to relabel the axes to Case 3 and vice versa. Likewise, if the dipolar interaction is strong enough to induce the alignment of the molecules along $p$ to form the $N_{U}$ phase, we need to relabel the axes so that $z \equiv p$ as in Case 2 .

\subsection{Self-consistent field equations}

We now formulate the internal energy per molecule as analogous to the pair potential defined in equation (??).

$$
U^{*}=-\frac{1}{2} g(\theta)\left(\mathcal{J}_{1}^{2}+2 \mathcal{J}_{2}^{2}\right)-\frac{1}{2} \kappa \mathcal{P}^{2},
$$

where we define the order parameters $\mathcal{J}_{i}, i=1,2$ as orientational averages of the functions $\left(J_{1}(\Omega), J_{2}(\Omega)\right)$ (see Eqs. ((??), (??)))

$$
\mathcal{J}_{i}=\int d \Omega f(\Omega) J_{i}(\Omega),
$$

with $i \in\{1,2\}$. Thus the analysis of our model requires only two order parameters, rather than the full complement of four. The internal energy in 
Eq. (??) is the consequence of the geometric mean approximation for the molecular interaction parameters [?, ?, ?], in addition to a dipolar interaction energy. We will address this issue in the Discussion. In the isotropic $(I)$ phase both order parameters are zero, in the $N_{U}$ phase, $\mathcal{J}_{1} \neq 0$, but $\mathcal{J}_{2}=0$, while in the $N_{B}$ phase neither order parameter is zero.

The orientational distribution function $f(\Omega)$ is derived by minimizing a Helmholtz free energy functional

$$
A^{*}=-T^{*} \int d \Omega f(\Omega) \log \left(8 \pi^{2} f(\Omega)\right)+U^{*} .
$$

The resulting equilibrium orientational distribution function $f(\Omega)$ is expressed in term of the potential of mean torque

$$
U^{*}(\Omega)=-g(\theta)\left(\mathcal{J}_{1} J_{1}(\Omega)+2 \mathcal{J}_{2} J_{2}(\Omega)\right)-\kappa \mathcal{P} R_{1}(\Omega),
$$

and is given by

$$
f(\Omega)=Q^{-1} \exp \left[-\frac{U^{*}(\Omega)}{T^{*}}\right],
$$

where the partition function $Q$ is defined in order to normalize $f(\Omega)$ :

$$
Q=\int d \Omega \exp \left[-\frac{U^{*}(\Omega)}{T^{*}}\right] .
$$

In Eq. (??), the dependence of $u$ and $\epsilon$ (see section ??) is given by $u=g(\theta) \epsilon$, analogous to the relation $\epsilon_{m}=g(\theta) \bar{\epsilon}$ in section ??. Thus in this model, there are three self-consistency equations, given by Eqs. (??) and (??). The phase stability of the system is determined from the equilibrium Helmholtz free energy

$$
A^{*}=-T^{*} \log Q-U^{*} .
$$

Note the apparent absence of the usual factor of $\frac{1}{2}$ in front of the energy $U^{*}$. The missing factor has been included in eq.(??).

\subsection{Method}

Rather than solve Eqs. (??) and (??) directly, we minimize the Helmholtz free energy in Eq. (??). The first derivatives of this equation are the selfconsistent Eqs. (??) and (??). We note that there are some subtle analytic points, which are addressed, for example by Katriel et al.[?]. Strictly speaking this procedure is not valid everywhere for finding minimizers of $A[f(\Omega)]$. We note that Eqs. (??) and (??) describe both the minima and the saddle 
points of the Helmholtz free energy in Eq. (??). Thus, in cases where Eq. (??) has saddle points, the method of minimising it is invalid and we have to resort to solving Eqs. (??) and (??) directly. However, the minimisation method is valid for finding stationary points. In the region of interest the method also suffices for determining the present quantities of interest.

The procedure determines the equilibrium order parameters $\left(\mathcal{P}, \mathcal{J}_{1}, \mathcal{J}_{2}\right)$ at a given temperature. The minimization uses the MATLAB function fmincon and iterates toward a solution using a quasi-Newton method. The phase transitions are found by determining $\left(\mathcal{P}, \mathcal{J}_{1}, \mathcal{J}_{2}\right)$, as a function of $T^{*}, \theta, \kappa$. The first-order transition is located when there is a discontinuous change in the order parameters as a function of $T^{*}$.

\section{Numerical results}

\subsection{Phase diagrams}

In Figs. 3 we show a representative set of four phase diagrams for increasing $\theta$ from $100^{\circ}$ to $130^{\circ}$. Each phase diagram shows a constant $\kappa$ slice in the $\left(\kappa-\theta-T^{*}\right)$ space. Our chosen values for $\theta$ and $\kappa$ are the same as those used in lattice simulations by Bates [?]. All topological configurations of phases that we find are shown in one of these examples. First-order and continuous transitions are plotted as continuous and broken lines, respectively.

First we review the zero dipolar interaction case $\kappa=0$ (see Fig. 3a) which has been discussed by several authors [?, ?, ?]. Here we use $N_{U}^{+}$ and $N_{U}^{-}$to denote the uniaxial nematic phases formed by the spontaneous alignment of the molecular axes $f$ and $e$, respectively. Thus $N_{U}^{+}$is a calamitic uniaxial nematic which exists between the $I$ and $N_{B}$ phases for $109.5^{\circ} \lesssim$ $\theta<180^{\circ}$ (equivalent to $0<\lambda<1 / \sqrt{6}$ ). On the other hand, $N_{U}^{-}$is discotic uniaxial nematic which exists between the $I$ and $N_{B}$ phases for $90^{\circ}<\theta \lesssim$ $109.5^{\circ}$ (equivalent to $1 / \sqrt{6}<\lambda<\sqrt{3 / 2}$ ). At the intersection of these two parameter ranges, namely $\theta=\theta_{c}=\cos ^{-1}(-1 / 3) \approx 109.5^{\circ}$ or $\lambda=1 / \sqrt{6}$ (see Fig. 2) (see also $[?, ?, ?, ?]$ ), the $I$ phase enters directly into the $N_{B}$ phase through a second-order phase transition at the Landau multicritical point. The stability of the non-polar biaxial nematic phase $N_{B}$ increases relative to that of the uniaxial nematic $N_{U}^{-}$on increasing $\theta$ from $100^{\circ}$ up to the Landau multicritical point at $\theta=\theta_{c}$. As $\theta$ increases away from $\theta_{c}$ the stability of $N_{B}$ decreases relative to that of $N_{U}^{+}$. Here, the ground state is always $N_{B}$. For $\theta \lesssim 100^{\circ}$ and $\theta \gtrsim 130^{\circ}$, the $N_{B}$ phase only intercedes at very low temperature. As $\theta$ approaches $\theta_{c}$ the $N_{B}-N_{U}$ phase boundary increases gradually in temperature. We note the sharp increase of the $N_{B}-N_{U}$ 


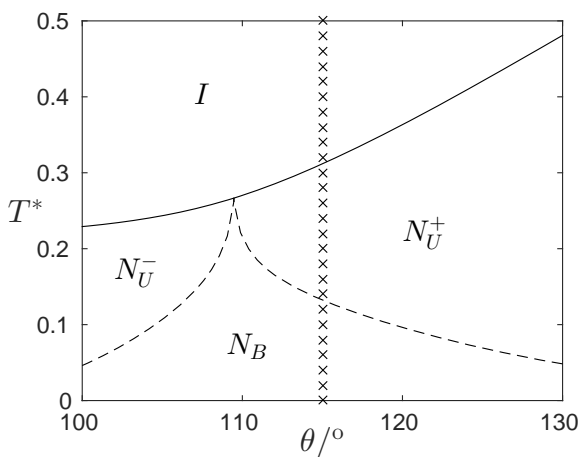

(a) $\kappa=0$

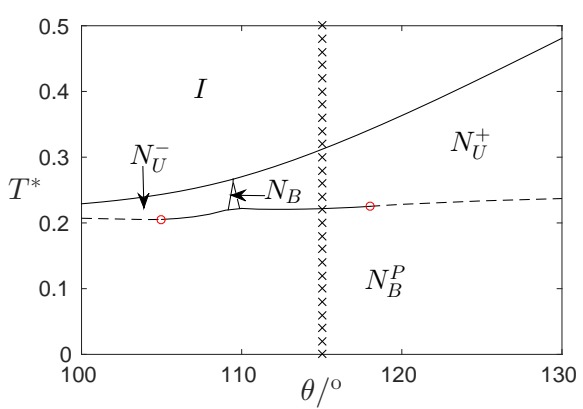

(c) $\kappa=0.5$

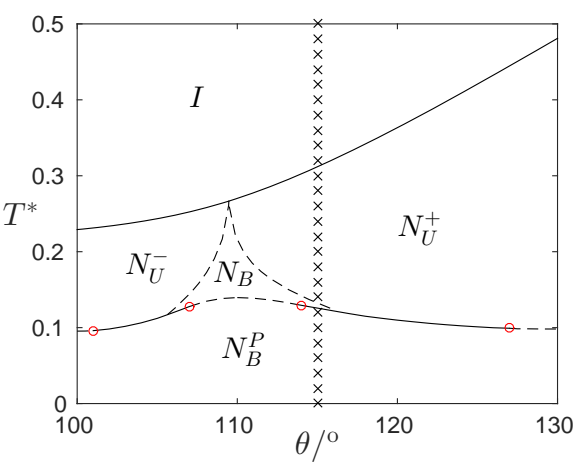

(b) $\kappa=0.2$

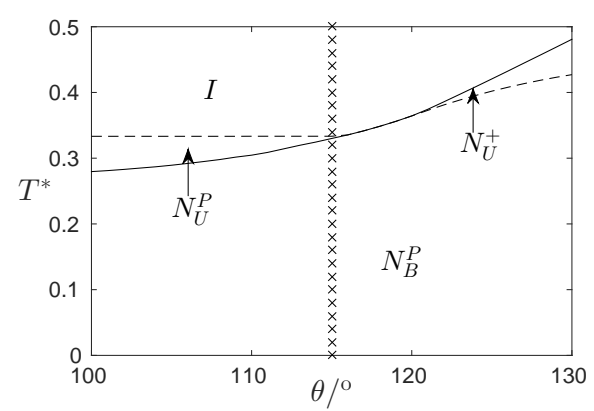

(d) $\kappa=1.0$

Figure 3: (Colour online) The phase diagrams as a function of the interarm angle $\theta$ and scaled temperature $T^{*}$, for representative values of the dipolar parameter $\kappa$. Continuous lines: first-order phase transitions; broken lines: second-order transitions; red circles: tricritical points. $I$ : isotropic phase. $N_{U}^{+}$: uniaxial nematic phase formed from aligning molecular axis $f . N_{U}^{-}$: uniaxial nematic phase formed from aligning molecular axis $e . N_{U}^{P}$ : uniaxial nematic phase formed by aligning molecular axis $p$ with dipolar ordering along $p . N_{B}$ : biaxial nematic phase. $N_{B}^{P}$ : biaxial nematic phase with polar ordering along molecular axis $p$. See Fig. 1 for definitions of the molecular axes. The vertical crosses indicate the temperature range over which the order parameters shown in Figs. 4 were calculated. 
transition temperature in the vicinity of $\theta_{c}$, indicating the sensitivity of the phase transition to $\theta$ at the Landau point. Then at the Landau multicritical point $\theta=\theta_{c}$, all phases coincide. We recall that for $\kappa=0$ there is an equivalent transformation of the transition temperatures between the two parameter spaces $0<\lambda<1 / \sqrt{6}$ and $1 / \sqrt{6}<\lambda<\sqrt{3 / 2}$ by changing the molecular axes from Case 1 to Case 3 (see Appendix ??) [?].

For $\kappa=0.2$ (see Fig. 3b), the higher temperature part of this diagram $\left(T^{*} \gtrsim 0.14\right)$ is unchanged from the $\kappa=0$ case, indicating that the dipolar interaction is sufficiently small that low temperatures are required to align the molecular dipoles. But now at sufficiently low temperatures a polar biaxial nematic phase $N_{B}^{P}$ is stabilized.

The ground state biaxial nematic phase is unsurprisingly polar, while for values of $\theta$ for which the $N_{B}$ phase requires very low temperatures, the $N_{U}$ phase yields to the $N_{B}^{P}$ phase. This picture seems generic for small positive $\kappa$. Although the $N_{U}$ and $N_{B}$ phases are retained at higher temperatures, the $N_{B}$ phase is now confined to a window around the Landau multicritical point. In the $\kappa=0.2$ case, this corresponds to $106^{\circ} \lesssim \theta \lesssim 117^{\circ}$. The tricritical points at $\theta \approx 101^{\circ}$ and $\theta \approx 127^{\circ}$ separate the first and continuous sections of the $N_{B}^{P}-N_{U}$ transition lines. Likewise, the tricritical points at $\theta \approx 107^{\circ}$ and $\theta \approx 114^{\circ}$ separate the first- and second- order sections of the $N_{B}^{P}-N_{B}$ transition line.

For $\kappa=0.5$ (see Fig. 3c), the stability regions of the non-polar nematic phases are smaller, as expected for a larger value of dipolar interaction. The $N_{B}$ stability has shrunk further, and is now just a sliver for $109^{\circ} \lesssim$ $\theta \lesssim 110^{\circ}$. Similar to the case for $\kappa=0.2$, the two tricritical points at $\theta \approx 105^{\circ}$ and $\theta \approx 118^{\circ}$ separate the first-order and continuous sections of the $N_{B}^{P}-N_{U}$ transition lines. The existence of the tricritical points in our model is interesting and resembles that found in the Sonnet-Virga-Durand approximation on the $N_{B}-N_{U}$ transition line [?].

In the final case we consider, $\kappa=1$ (see Fig. 3d). Here, a new uniaxial phase $N_{U}^{P}$ appears which is formed by aligning the molecular dipoles $\mathbf{p}$ parallel. The polar phases $N_{U}^{P}$ and $N_{B}^{P}$ replace the non-polar $N_{U}$ phase over most of its range. Both the $N_{B}$ phase and the Landau multicritical point disappear. Instead there is a line of first-order $N_{B}^{P}-I$ transitions for $116^{\circ} \lesssim \theta \lesssim 121^{\circ}$

\subsection{Order parameters of the arms}

For the three cases $\kappa=0,0.2,0.5$, shown in Figs. 4a, $4 \mathrm{~b}$ and $4 \mathrm{c}$, respectively, the $I-N_{U}$ phase transition occurs at $T^{*} \approx 0.31$ where $S^{A A} \neq 0$ and 
$S^{X X}=S^{Y Y}=-\frac{1}{2} S^{Z Z}$. For apolar or small dipolar interaction $\kappa=0,0.2$, the $N_{U}-N_{B}$ transition takes place at a common temperature, $T^{*} \approx 0.13$, where $S^{X X} \neq S^{Y Y} \neq S^{Z Z}$ and $S^{Z Z}$ is the largest component. This feature seems to be generic for $0 \leq \kappa \lesssim 0.2$.

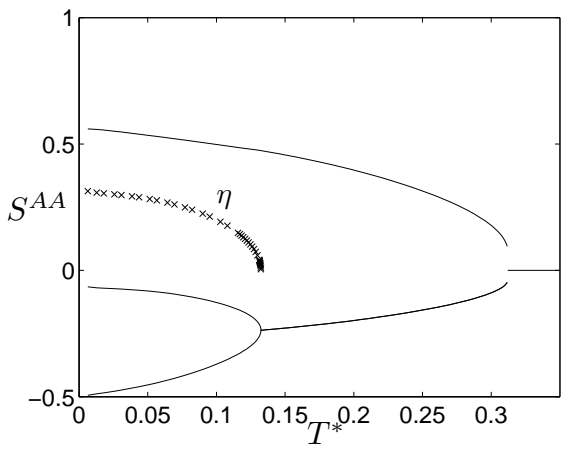

(a) $\kappa=0$

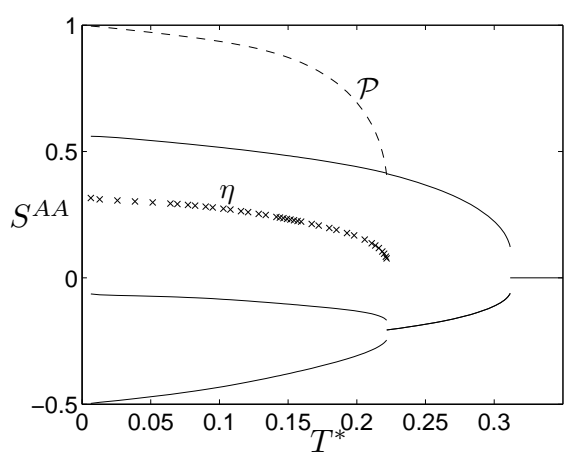

(c) $\kappa=0.5$

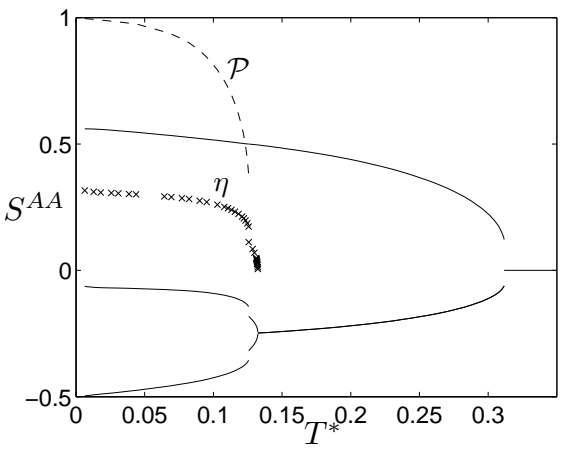

(b) $\kappa=0.2$

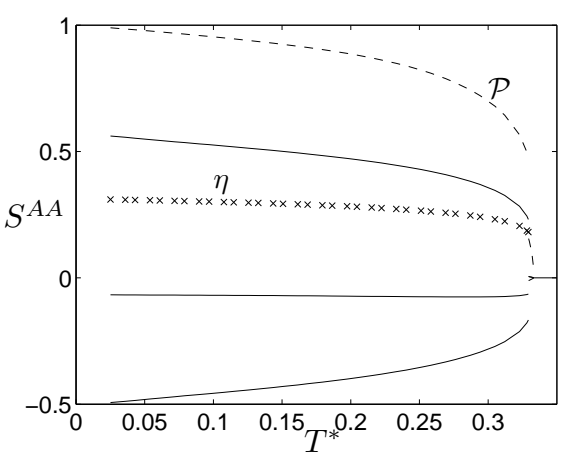

(d) $\kappa=1.0$

Figure 4: The temperature dependence of the order parameter tensor components of the rod-like arm $S^{X X}, S^{Y Y}$ and $S^{Z Z}$ (continuous lines), the polar order parameter $\mathcal{P}$ (broken line) and the relative biaxiality parameter $\eta$ (crosses) for interarm angle $\theta=115^{\circ}$. We note that the limiting values of $\eta$ are the same for all of these systems; this limit is related to the inter-arm angle by $\eta=\frac{3 \cos ^{2} \theta / 2}{2-3 \cos ^{2} \theta / 2}$.

To make more detailed comparison with simulations by Bates [?], we calculate the three tensorial components $S^{A A}, A=X, Y, Z$ for the molecular arms for $\theta=115^{\circ}$ (see Appendix ??). Their values are shown in Figs. 4. 
We also plot the polar order parameter $\mathcal{P}$ as broken lines. Here, first-order transitions are clearly seen as discontinuous changes in the order parameters. In addition, we also show the relative biaxiality parameter $\eta$ as crosses, where

$$
\eta=\frac{S^{X X}-S^{Y Y}}{S^{Z Z} \sqrt{6}}
$$

As we see in Figs. 3, the ground state for non-zero dipole cases is the $N_{B}^{P}$ phase. For $\kappa=0.2$ (see Fig. $3 \mathrm{~b}$ ), the $N_{B}^{P}-N_{B}$ transition occurs at $T^{*} \approx 0.125$. For stronger dipole $\kappa=0.5$ (see Fig. 3c), the $N_{B}^{P}$ phase is formed directly from the $N_{U}^{+/-}$phase at $T^{*} \approx 0.23$.

In the case with the strongest dipolar interaction that we study, $\kappa=1$ (see Fig. 4d), the polar $N_{U}^{P}$ phase is formed directly from the isotropic phase although this phase occurs over a tiny temperature range at $T^{*} \approx 0.37$. In this phase, $S^{X X}=S^{Z Z}=-\frac{1}{2} S^{Y Y}$. At a slightly lower temperature, the first-order $N_{B}^{P}-N_{U}^{P}$ phase transition occurs. In the $N_{B}^{P}$ phase, $S^{X X} \neq$ $S^{Y Y} \neq S^{Z Z}$ and $S^{Y Y}$ is now the largest component.

\section{Discussion and Conclusions}

This paper was prompted by an apparent disagreement between theory and experiment. A small nematic phase biaxiality was found in a system of bent-core molecules in experiments by Madsen et al.[?, ?]. However it was noted by Luckhurst [?] that the onset of the $N_{B}$ phase occurs at a much higher temperature than the theoretical prediction for bent-core molecules. It was suggested that this might be due to a large transverse electrostatic dipole present in the bent-core molecules [?, ?]. However, if this is the case, the transverse dipole will stabilize the alignment of the short molecular axis, and thus the resulting biaxial nematic phase might be polar.

To test this hypothesis, we have developed a simplified two-parameter molecular field theory for polar biaxial nematics formed from rigid V-shaped bent-core molecules. The theory extends the standard model for uniaxial and biaxial nematics to allow for polar ordering of a minor axis. We have used the same values for the biaxiality and dipole parameters as the lattice Monte Carlo simulations by Bates [?].

We find phase diagrams in qualitative agreement with the simulations. Firstly, the stability of the biaxial nematic phase increases upon increasing the dipole strength $\kappa$. Secondly, for a large dipole strength, $\kappa=1$, the Landau multicritical point in a non-polar system develops into a line of first-order biaxial nematic-to-isotropic transitions. 
However, we also find extra features not reported in the simulations [?]. Firstly, for positive $\kappa$, the ground state is always a polar biaxial nematic $N_{B}^{P}$. For zero or small $\kappa(0,0.2,0.5)$, the $N_{B}^{P}$ phase is formed from one of the non-polar phases $N_{U}$ or $N_{B}$. For the large dipole strength, $\kappa=1$, the Landau multicritical point develops into a line of $N_{B}^{P}-I$ transitions. Secondly, the uniaxial nematic phase that we found for $\kappa=1$ is in fact a polar uniaxial nematic phase $N_{U}^{P}$; this is in the same region as the $N_{U}$ phase found in the simulations by Bates [?]. This phase is formed by aligning only the molecular short axis along the dipole moment. Our findings are consistent with the remark by Ghoshal et al.[?] that the Heisenberg form for the dipolar interaction can stabilize polar phases.

Our result for the $N_{B}^{P}-I$ transition line also provides an explanation for the disagreement between theory and experiment. In the presence of a strong dipolar interaction, a system formed from relatively low biaxiality bentcore molecules is still able to form a biaxial nematic phase directly below the isotropic phase due to the formation of the $N_{B}^{P}$ phase. We note that another explanation is also possible. The hypothesis that phase biaxiality is stabilized by electrostatic dipolar interaction proposed by Madsen et al.[?] has been confirmed by previous simulation studies by Peláez and Wilson [?], Bates [?], Ghoshal et al.[?] and Osipov and Pająk [?]. However, the atomistic simulations by Peláez and Wilson [?] found local polar-order domains in the biaxial phase while studies by Bates [?], Ghoshal et al.[?] and Osipov and Pająk [?] did not find any polar phases. These simulation results suggest another explanation for the stabilisation of the biaxial phase, namely, the consequence of local ordering of the molecular dipoles. Indeed, the two-sitecluster study by Osipov and Pająk [?] has demonstrated that there are strong polar correlations between electrostatic and steric dipoles of neighbouring bent-core molecules both in $N_{U}$ and $N_{B}$.

To compare our results with the experiment by Madsen et al.[?] directly, we plot the phase diagram for a constant molecular biaxiality slice at the interarm angle of $140^{\circ}$ in Fig. 5 (the value estimated for their bent-core compounds). The compounds in their experiment, namely ODBP-Ph-OC 12 and $\mathrm{ODBP}-\mathrm{Ph}-\mathrm{C}_{7}$, experience a direct biaxial nematic-to-isotropic phase transition. From Fig. 5, we see that this happens for the relative dipole strength $1.55 \lesssim \kappa \lesssim 1.75$. We can also compare our values for the relative phase biaxiality given in Eq. (??) for $\theta=140^{\circ}$ and $\kappa=1.6$ with the NMR experiment by Madsen et al.[?]. In the experiment, the $N_{B}-I$ transition temperatures for ODBP-Ph-OC 12 and ODBP-Ph- $\mathrm{C}_{7}$ are $477 \mathrm{~K}$ and $495 \mathrm{~K}$, respectively. In addition, the given experimental values for $\eta$ for these systems at $463 K$ and $447 K$ are $0.02 \pm 0.02$ and $0.11 \pm 0.02$, respectively. Thus we 


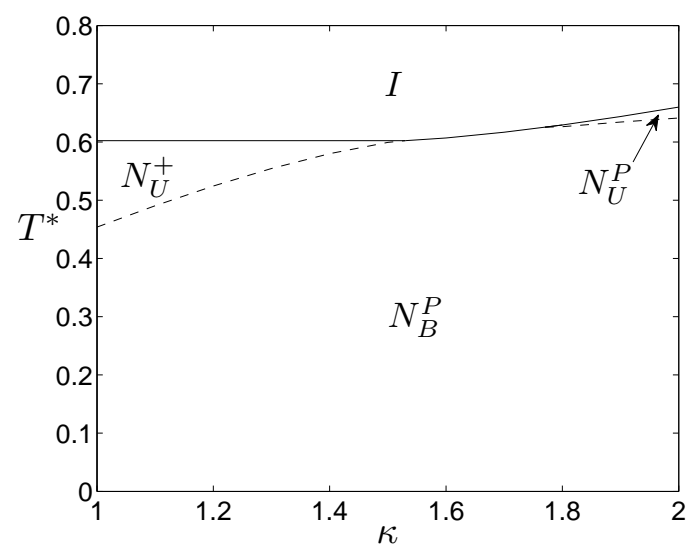

Figure 5: The phase diagram as a function of relative dipolar strength $\kappa$ and scaled temperature $T^{*}$, for the interarm angle $\theta=140^{\circ}$. Continuous lines: first-order phase transitions; broken lines: continuous (or secondorder) transitions.

calculate the values for $\eta$ at $463 / 477 T_{N_{B}-I}$ and $447 / 495 T_{N_{B}-I}$ and obtain 0.041 and 0.046 , respectively. We conclude that these values are of the same order of magnitude as the experimental values with differintg by factors of 2 and 4 . Therefore within our hypothesis, the polar order with $\mathcal{P}=0.5$ (see Fig. 4d) for these systems induces only a small phase biaxiality. This suggests that this polar induction of phase biaxiality can give weak phase biaxiality whereas for Freiser the quadrupolar terms [?] gives larger values.

We have found two biaxial nematic phases with point group symmetries $D_{2 h}$ (nonpolar biaxial phase) and $C_{2 v}$ (polar biaxial phase). The point groups symmetry $D_{2 h}$ has an identity element, three orthogonal two-fold symmetry axes, an inversion center and three orthogonal reflection symmetry planes. When the dipoles align in the $N_{B}^{P}$ phase, two symmetry axes, one symmetry plane and the inversion center are broken, thus the phase symmetry is reduced to $C_{2 v}$. We note that the theoretial analysis by Karahaliou, Vanakaras and Photinos [?] has suggested that the biaxial nematic phases formed from bent-core molecules might have the point group symmetry $C_{2 h}$. This point group symmetry has an identity element, one two-fold symmetry axis, one reflection symmetry plane and an inversion center. Indeed one of the bent-core compounds which forms the biaxial nematic phase [?, ?] has an asymmetric dipole moment which points away from the bent-core angu- 
lar bisector, but is still coplanar with the molecular arms. This bent-core molecule has $C_{s}$ point groups symmetry with an identity element and one reflection plane. Thus in this case we can speculate that three $N_{B}$ phases with different point group symmetry. When the dipolar interactions are weak, the dipoles do not align, the $N_{B}$ phase with $D_{2 h}$ symmetry can be formed. When the dipoles align in anti-parallel configuration, the symmetry of the $N_{B}$ phase is reduced to $C_{2 h}$. Finally, in the $N_{B}^{P}$ phase, the dipoles align in parallel configuration, the two-fold rotation axis and the inversion center in the $N_{B}$ phase with $C_{2 h}$ symmetry are destroyed and the symmetry is reduced to $C_{s}$. The Monte-Carlo simulations by Ye, Merlitz and $\mathrm{Wu}$ [?] for bent-core molecules with asymmetric dipoles has demonstrated that for discotic bent-core molecules (with $90^{\circ} \leq \theta \lesssim 109.5^{\circ}$ ), as the dipole deviates from the bisector, the temperature range of the $N_{B}$ phase increases whereas the temperature range of the $N_{U}$ phase decreases. In this case we may expect that a $N_{B}$ phase of low symmetry $\left(C_{2 h}\right.$ or $\left.C_{s}\right)$ is formed and is stabilized for a longer temperature range than the $N_{B}$ phase with $D_{2 h}$ symmetry. This is in accordance with a previous study of the molecular field theory for nematic phases formed from molecules with $C_{2 h}$ point group symmetry [?] that, the $N_{B}$ phase with the lower symmetry $C_{2 h}$ can exist for a longer temperature range compared to the $N_{B}$ phase with $D_{2 h}$ symmetry.

We now discuss the approximations that we have made. Firstly, we assume that a bent-core molecule has a rigid V-shape and interacts with another via a linear combination of the uniaxial interaction of each arm. In addition, the uniaxial interaction of molecular arms has the same form as in the Maier-Saupe theory which depends on the relative orientations of the molecular arms. Our assumptions are partly for simplicity but also have a justification based on the success of the Maier-Saupe theory. In addition, our form for the molecular interactions also agree with the so-called geometric mean approximation [?, ?, ?]. This uses the Berthelot combining rule to reduce the number of molecular biaxiality parameters from 2 to 1 .

The Heisenberg model for the dipolar interaction in Eq. (??) has an isotropic distribution for the intermolecular vector and favors parallel alignment of neighbouring dipoles for $\kappa>0$. We discuss the applicability of such a model later, but note here that other authors have used more realistic models for the dipolar interaction both in density functional theory [?], lattice Monte Carlo simulations [?] and two-site cluster theory [?]. These studies include the effect of short-range order which, of necessity, do not appear in our molecular field theory. In neither case do polar phases seem to occur, although the existence of a dipolar interaction nevertheless does stabilize the biaxial nematic phase. The first study [?] found a shift in the 
Landau multicritical point as the dipole strength is increased. The second study [?] found similar qualitative features as our results. These are the stabilisation of the biaxial nematic phase with increasing dipolar strength and the development of the Landau multicritical point into a line of firstorder biaxial nematic-to-isotropic phase transitions. Moreover, in the biaxial phase, there is an equal number of dipoles aligning both parallel and antiparallel, and thus on average the polar order parameter cancels. The third study [?] found that both electrostatic interaction and polar steric effect increase the stability of the $N_{B}$ phase. However, we note that the model by Ghoshal [?] and Osipov and Pająk [?] assumes a linear combination of the first- and second-rank interactions. Other studies by Palffy-Muhoray et al.[?] and Sonnet et al.[?, ?] have included non-linear combinations of firstand second-rank interactions. Detailed studies of these models for biaxial bent-core nematics may well find that the $N_{B}^{P}$ phase is stabilized in the ground state.

Finally, we focus on the key approximation of our model, which involves replacing the rather complex but realistic electrostatic dipolar interaction by a directionally-independent Heisenberg form. At first sight this approximation seems rather far-fetched since the dipolar interaction energy in Eq. (??) vanishes as we take the average of the pair potential over all orientations of the intermolecular vector. Our picture is that the apparent Heisenberg form results from a combination of steric and electrostatic effects. In this context we note that studies by Sonnet et al.[?, ?] have shown that the polar steric interaction of bent-core molecules favours local anti-parallel configurations, thus weakening the effect of electrostatic dipolar interaction. In future work we shall use a more realistic dipolar form which includes both electrostatic and steric effects to test the hypothesis of the current paper, and to find a balance between these two effects that may stabilize the $N_{B}^{P}$ phase. One possibility is to use a simple model developed recently by Osipov and Pająk [?] which also has a more realistic description of the dipolar interaction. In this model, each molecule is composed of three coplanar spheres and a transverse electrostatic dipole. The angle formed by connecting one of the centres of the spheres to the other two reflects the molecular biaxiality. The molecular interaction consists of an electrostatic dipole-dipole potential and a Lennard-Jones potential between spheres of different molecules. Thus this simple model can be used to study the influence of both electrostatic and steric effects on the stability of the polar nematic phases. 


\section{Acknowledgments}

TBTT acknowledges financial support from the School of Mathematical Sciences, University of Southampton, through a School Ph.D. Studentship. TBTT thanks Prof. E.G. Virga for a helpful discussion. The final parts of this research were carried out while TJS and TBTT were participants in the "Mathematics of Liquid Crystals" programme at the Isaac Newton Institute for Mathematical Sciences, University of Cambridge (January-July 2013). They thank the organisers of this programme for the invitation to participate, and the Director of the Institute for his hospitality during the course of the programme.

\section{A Molecular anisotropy and biaxiality in different coordinate systems}

In this Appendix we show how to apply Eq. (??)

$$
\begin{aligned}
& U_{(2)}^{*}(\Omega)=\frac{U_{(2)}(\Omega)}{\bar{\epsilon}} \\
& =-\sum_{i, j=1,2} P_{2}\left(\cos \phi_{i j}^{A B}\right)-\kappa R_{1}(\Omega),
\end{aligned}
$$

to three different molecular axis systems which are discussed in section ??. We recall that $\Omega$ denotes the three Euler angles required to transform the molecular axes of molecule $A$ into those of molecule $B . \phi_{i j}^{A B}$ denotes the two Euler angles required to transform the long axis of arm $i$ of molecule $A$ into that of arm $j$ of molecule $B$. Thus $\phi_{i j}^{A B}$ is the result of three successive rotations $\left(\omega_{i}^{A-1}, \Omega, \omega_{j}^{B}\right)$ where $\omega_{i}^{A}$ denotes the two Euler angles required to transform the molecular axis $z$ of molecule A into the long axis of the arm $i$ of molecule $A$ (we will consider three cases with different locations of $z$ ). Likewise, $\omega_{j}^{B}$ denotes the two Euler angles required to transform the molecular axis $z$ of molecule $B$ into the long axis of the arm $j$ of molecule $B$. For convenience, we denote $\omega^{\prime}$ as the result of two successive rotations $\left(\Omega, \omega_{j}^{B}\right)$. Thus $\phi_{i j}^{A B}$ is the result of two successive rotations $\left(\omega_{i}^{A^{-1}}, \omega^{\prime}\right)$. In order to transform $P_{2}\left(\cos \phi_{i j}^{A B}\right)$ into the coordinate of $\Omega$, we use the modified spherical harmonic notations $C_{L m}(\omega)$, these are also known as Racah spherical harmonics [?]. Their relations to the standard spherical harmonics $Y_{L m}(\omega)$ are given in Eq. A.11, p. 445 [?]. Using the spherical harmonic addition 
theorem (see Eq. A.33, p. 451 [?]) and Eq. A.3, p. 442 [?] we find

$$
\begin{aligned}
P_{2}\left(\cos \phi_{i j}^{A B}\right) & =\sum C_{2 p}\left(\omega_{i}^{A}\right)^{*} C_{2 p}\left(\omega^{\prime}\right) \\
& =\sum(-1)^{p} C_{2-p}\left(\omega_{i}^{A}\right) C_{2 p}\left(\omega^{\prime}\right),
\end{aligned}
$$

since $\phi_{i j}^{A B}$ is the angle between the vectors $\omega_{i}^{A}$ and $\omega^{\prime}$. Using transformation under rotation (Eq. A.41, p. 452 [?]) we obtain

$$
C_{2 p}\left(\omega^{\prime}\right)=\sum D_{n p}^{2}(\Omega) C_{2 n}\left(\omega_{j}^{B}\right) .
$$

Thus

$$
P_{2}\left(\cos \phi_{i j}^{A B}\right)=\sum(-1)^{p} C_{2-p}\left(\omega_{i}^{A}\right) C_{2 n}\left(\omega_{j}^{B}\right) D_{n p}^{2}(\Omega)
$$

\section{A.1 Case 1: $(p, e, f) \equiv(x, y, z)$ rod-like molecule}

. We have

$$
\omega_{12}^{A B}=\left(\beta_{12}^{A B}, \gamma_{12}^{A B}\right)=(\pi / 2 \pm \theta / 2,0)
$$

Thus,

$$
\begin{gathered}
C_{20}\left(\omega_{12}^{A B}\right)=\frac{1}{4}(1-3 \cos \theta) ; \\
C_{2 \pm 1}\left(\omega_{12}^{A B}\right)=0 ; \\
C_{2 \pm 2}\left(\omega_{12}^{A B}\right)=\frac{1}{4} \sqrt{\frac{3}{2}}(1+\cos \theta) .
\end{gathered}
$$

By substituting Eqs. (??, ??, ??, ??) into Eq. (??), we obtain Eqs. (??)(a) and $(? ?)(b)$.

\section{A.2 Case 2: $(p, e, f) \equiv(z, y, x)$ dipolar molecule}

. We have

$$
\omega_{12}^{A B}=\left(\beta_{12}^{A B}, \gamma_{12}^{A B}\right)=( \pm \theta / 2,0)
$$

Thus,

$$
\begin{gathered}
C_{20}\left(\omega_{12}^{A B}\right)=\frac{1}{4}(1+3 \cos \theta) ; \\
C_{2 \pm 1}\left(\omega_{12}^{A B}\right)=0 ; \\
C_{2 \pm 2}\left(\omega_{12}^{A B}\right)=\frac{1}{4} \sqrt{\frac{3}{2}}(\cos \theta-1) .
\end{gathered}
$$

By substituting Eqs. (??, ??, ??, ??) into Eq. (??), we obtain Eqs. (??)(a) and $(? ?)(b)$. 


\section{A.3 Case 3: $(p, e, f) \equiv(y, z, x)$ disc-like molecule}

. We have

$$
\omega_{12}^{A B}=\left(\pi / 2, \gamma_{12}^{A B}\right)=(\pi / 2, \pm(\pi / 2-\theta / 2)) .
$$

Thus,

$$
\begin{gathered}
C_{20}\left(\omega_{12}^{A B}\right)=1 ; \\
C_{2 \pm 1}\left(\omega_{12}^{A B}\right)=0 ; \\
C_{2 \pm 2}\left(\omega_{12}^{A B}\right)=-\frac{1}{4} \sqrt{\frac{3}{2}} \cos \theta .
\end{gathered}
$$

By substituting Eqs. (??, ??, ??, ??) into Eq. (??), we obtain Eqs. (??)(a) and $(? ?)(\mathrm{b})$.

We note that previously cases 1 and 2 were analysed by Bates and Luckhurst [?] using the separability approximation for the interaction tensors. Here we have provided an alternative derivation for all three cases which does not require this approximation.

\section{B Order parameters of the arms in Cartesian co- ordinates}

In this Appendix we discuss the calculations for the order parameters of the arms in Cartesian coordinates $S^{A A}, A=X, Y, Z$ in Section ??. The first step is to calculate the Cartesian order parameters for the molecule. These are given by

$$
S_{a a}^{A A}=\frac{1}{2}\left(3\left\langle l_{A a}^{2}\right\rangle-1\right) ; a=x, y, z ; A=X, Y, Z .
$$

Here, $l_{A a}$ denotes the direction cosines between the reference axes $A$ and the molecular axes $a$. The dependence of the components of $l_{A a}$ on the Euler 
angles is given by

$$
\begin{aligned}
& l_{X x}=-\sin \alpha \sin \gamma+\cos \beta \cos \alpha \cos \gamma, \\
& l_{X y}=-\sin \alpha \cos \gamma-\cos \beta \cos \alpha \sin \gamma, \\
& l_{X z}=\sin \beta \cos \alpha, \\
& l_{Y x}=\cos \alpha \sin \gamma+\cos \beta \sin \alpha \cos \gamma, \\
& l_{Y y}=\cos \alpha \cos \gamma-\cos \beta \sin \alpha \sin \gamma, \\
& l_{Y z}=\cos \beta \sin \alpha, \\
& l_{Z x}=-\sin \beta \cos \gamma, \\
& l_{Z y}=\sin \beta \sin \gamma, \\
& l_{Z z}=\cos \beta .
\end{aligned}
$$

The next step is to transform from the molecular coordinate axes to the axis $s$ along an arm (see Fig. 1) by

$$
S^{A A}=\sum_{b}\left(l_{s b}\right)^{2} S_{b b}^{A A} ; A=X, Y, Z ; b=x, y, z .
$$

For our calculation, we consider two cases

- Case 1: $(p, e, f) \equiv(x, y, z)$

$$
S^{A A}=\cos ^{2}(\theta / 2) S_{x x}^{A A}+\sin ^{2}(\theta / 2) S_{z z}^{A A} .
$$

- Case 2: $(p, e, f) \equiv(z, y, x)$

$$
S^{A A}=\cos ^{2}(\theta / 2) S_{z z}^{A A}+\sin ^{2}(\theta / 2) S_{x x}^{A A} .
$$

